\title{
Low-Load Bench Press Training to Fatigue Results in Muscle Hypertrophy Similar to High-Load Bench Press Training
}

\author{
Riki Ogasawara $^{1,2}$, Jeremy P. Loenneke ${ }^{3}$, Robert S. Thiebaud ${ }^{3}$, Takashi Abe ${ }^{1,4}$ \\ ${ }^{1}$ Graduate School of Frontier Sciences, University of Tokyo, Kashiwa, Japan; ${ }^{2}$ College of Sport and Health Science, Ritsumeikan \\ University, Kusatsu, Japan; ${ }^{3}$ Department of Health and Exercise Science, University of Oklahoma, Norman, USA; ${ }^{4}$ Department of \\ Health, Exercise Science, \& Recreation Management, University of Mississippi, Oxford, USA. \\ Email: t12abe@gmail.com
}

Received December $20^{\text {th }}$, 2012; revised January $20^{\text {th }}$, 2013; accepted January $27^{\text {th }}, 2013$

\begin{abstract}
The purpose of this study was to determine whether the training responses observed with low-load resistance exercise to volitional fatigue translates into significant muscle hypertrophy, and compare that response to high-load resistance training. Nine previously untrained men (aged 25 [SD 3] years at the beginning of the study, standing height 1.73 [SD 0.07] m, body mass 68.9 [SD 8.1] kg) completed 6 weeks of high load-resistance training (HL-RT) (75\% of one repetition maximal [1RM], 3-sets, 3x/wk) followed by 12 months of detraining. Following this, subjects completed 6 weeks of low load-resistance training (LL-RT) to volitional fatigue (30\% 1 RM, 4 sets, 3x/wk). Increases $(p<0.05)$ in magnetic resonance imaging-measured triceps brachii and pectoralis major muscle cross-sectional areas were similar for both HL-RT (11.9\% and 17.6\%, respectively) and LL-RT (9.8\% and 21.1\%, respectively). In addition, both groups increased $(p<0.05) 1 \mathrm{RM}$ and maximal elbow extension strength following training; however, the percent increases in 1 RM ( $8.6 \%$ vs. $21.0 \%)$ and elbow extension strength $(6.5 \%$ vs. $13.9 \%)$ were significantly $(p<0.05)$ lower with LL-RT. Both protocols elicited similar increases in muscle cross-sectional area, however differences were observed in strength. An explanation of the smaller relative increases in strength may be due to the fact that detraining after HL-RT did not cause strength values to return to baseline levels thereby producing smaller changes in strength. In addition, the results may also suggest that the consistent practice of lifting a heavy load is necessary to maximize gains in muscular strength of the trained movement. These results demonstrate that significant muscle hypertrophy can occur without high-load resistance training and suggests that the focus on percentage of external load as the important deciding factor on muscle hypertrophy is too simplistic and inappropriate.
\end{abstract}

Keywords: Bench Press; Training Intensity; Muscle CSA; MRI; Strength

\section{Introduction}

As a muscle is overloaded from increased mechanical work, the added stress increases skeletal muscle amino acid transporter expression [1], which in turn enhances the synthesis of the contractile proteins, actin and myosin [2]. These acute positive balances between muscle protein synthesis (MPS) and muscle protein breakdown (MPB) lead to skeletal muscle hypertrophy over time which occurs from both an increase in the thickness and number of myofibrils [see molecular pathway review by Adams [3]. Although skeletal muscle hypertrophy occurs in both slow twitch (ST) and fast twitch (FT) fibers, the latter has the greatest potential for growth [4]. Therefore it is been hypothesized that skeletal muscle hypertrophy can occur independent of exercise load, as long as FT fibers are activated [5,6].

Conventional thought is that at least $70 \%$ of one's repetition maximum (1 RM) must be lifted repeatedly to observe a meaningful increase in muscular size [7]. However, acute molecular research indicates that external exercise load may be of less importance when adequate volume of resistance exercise is completed. To illustrate, when four sets of resistance exercise was performed at $30 \% 1$ RM to volitional fatigue, myofibril MPS was elevated to the same level as $90 \% 1 \mathrm{RM}$ to volitional fatigue (not work matched) [8]. This is contrary to what has commonly been reported in the literature which states that training to volitional fatigue is not an effective stimulus unless a sufficient external load as defined by percentage of $1 \mathrm{RM}(\sim 80 \% 1 \mathrm{RM})$ is lifted. The common thought has always been that higher repetition training 
cannot produce a stress that is adequate enough to recruit and fatigue the highest threshold motor units [9].

Interestingly, Campos et al. [10] provide the only evidence to date that resistance exercise to volitional fatigue at higher loads is more effective than training at lower loads for skeletal muscle hypertrophy (4 sets 3 - 4 RM vs. 2 sets 20 - $28 \mathrm{RM}$ ). However, using the identical methods of Campos et al. [10], Leger et al. [11] observed significant increases in muscle hypertrophy, muscular strength, and endurance independent of the external load lifted. One possible reason for the difference could be due to the older less active subjects used in latter study (36 vs 22 yrs). In addition, the volume of exercise (2 sets) may have been inadequate to recruit the higher threshold motor units in the younger more active subjects used in the Campos et al. [10] paper.

The aforementioned evidence has led to the formation of the metabolite/volume threshold theory [5]. This theory states that, assuming an adequate exercise volume is achieved, the recruitment of FT fibers appears to be the large driving force of skeletal muscle hypertrophy whereas the external load lifted and systemic endogenous hormone elevations may not be as important as previously thought $[12,13]$. Much of this theory was based on acute myofibril MPS and it is acknowledged that although these acute studies are hypothesized to be predictive of chronic adaptations, they are not definitive as incongruences may exist between the acute and chronic changes following resistance training $[14,15]$. Therefore, the purpose of this study was to determine whether the training responses observed with low-load resistance exercise to volitional fatigue translates into significant muscle hypertrophy, and compare that response to high-load resistance training. Low load knee extensor exercise to fatigue has shown that muscle hypertrophy (whole muscle and fiber level) occurs at levels similar to higher loads [16], however it is currently unknown whether this is also true for upper body resistance exercise. Bench press is one of the major exercises for developing the upper body, however, very few studies report muscle size changes in the chest and upper arm following a single mode of high-load bench press training $[17,18]$. In the present study, a within subject experimental design was chosen to reduce biological variability. Further, due to possible differences in systemic endogenous hormones with each loading scheme and the cross-training neural adaptations associated with a unilateral training model [19], each subject completed both exercise protocols separated by over a year (12 months). All subjects began with high-load resistance training as this design also allowed us to investigate the muscle size and strength changes to one year of detraining with traditional high load exercise. Although the order of training was not randomized, it increased our statistical power to investi- gate at least one of our purposes with the possibility of a poor attrition rate with such a long investigation. We hypothesized that similar increases in muscle hypertrophy would be observed with both protocols, independent of the external load lifted.

\section{Material and Methods}

\subsection{Subjects}

Nine previously untrained young men (aged 25 [SD 3] years at the beginning of the study) volunteered to participate in two different 6-week resistance training protocols separated by 12 months (Table 1). In the first training protocol, all subjects performed high-load (75\% of $1 \mathrm{RM}$ ) resistance exercise. Twelve months after the end of the first training protocol, the subjects performed the second resistance training program with low-loads (30\% of $1 \mathrm{RM}$ ). None of the subjects performed resistance training as well as aerobic-type training for at least 9 months prior to the start of the second training protocol. Subjects were instructed to maintain their usual dietary regimen throughout the study. All subjects were informed of the procedures, risks, and benefits and signed an informed consent document. The study was conducted according to the Declaration of Helsinki and was approved by the Ethics Committee for Human Experiments at The University of Tokyo, Japan.

\subsection{Resistance Training Protocol}

Free-weight bench press exercise was performed 3 days per week (Monday, Wednesday, Friday) in both the high-load (HL-RT) as well as the low-load (LL-RT) resistance training protocol. The exercise session in the HL-RT consisted of 3 sets ( 3 min rest between sets) of 10 reps at $75 \%$ of $1 \mathrm{RM}$, while the exercise session with LLRT consisted of 4 sets (3 min rest between sets) of bench press exercise until volitional fatigue at $30 \%$ of $1 \mathrm{RM}$. During HL-RT and LL-RT exercise sessions, the velocities of the eccentric and concentric movements were standardized to approximately 2-second (eccentric $\sim 1 \mathrm{~s}$, concentric $\sim 1 \mathrm{~s}$ ) using a metronome. During the latter repetitions for the HL-RT, velocity decreased to $~ 2$

Table 1. Physical characteristics of the subjects.

\begin{tabular}{cccc}
\hline & Height & Body mass & Body mass index \\
\hline & $(\mathrm{m})$ & $(\mathrm{kg})$ & $\left(\mathrm{kg} / \mathrm{m}^{2}\right)$ \\
HL-RT pre (0.07) & 1.73 & $68.9(8.1)$ & $23.0(2.8)$ \\
HL-RT post & & $69.5(8.5)^{*}$ & $23.2(2.8)$ \\
LL-RT pre (0.07) & 1.74 & $68.8(8.0)$ & $22.9(2.8)$ \\
LL-RT post & & $69.4(7.9)^{*}$ & $23.1(2.5)$ \\
\hline
\end{tabular}

HL-RT, high-load resistance training; LL-RT, low-load resistance training; ${ }^{*} p<0.05$, pre vs. post. 
sec per muscle action. Training load was adjusted to the new 1RM determined at 3 weeks in both training protocols. For the HL-RT, if subjects were able to perform 12 repetitions or more during a training session, the training load was increased $\sim 5 \%$ for the next training session. To ensure adequate training load, all training sessions were surveyed and supervised by trained personnel. All subjects successfully completed every training session.

\subsection{Measurements Schedule}

Subjects testing took place before the start of the study (pre) and 3 - 4 days after (post) the 6-week training period. The magnetic resonance imaging (MRI) measurement was obtained between 16:00 and 19:00 hours. The strength measurement was determined on the same day or the following day after the MRI measurement. All measurements were balanced for the time of day.

\subsection{Strength Measurement}

All subjects completed 2 - 3 familiarization sessions to receive instruction on proper technique and to practice the $1 \mathrm{RM}$ and maximal voluntary isometric strength (MVC) tests. The 1RM was assessed with the freeweight bench press exercise. The $1 \mathrm{RM}$ was determined by progressively increasing the weight lifted until the subject failed to lift the weight through a complete range of motion. Usually 5 trials were required to complete a 1 $\mathrm{RM}$ test. Adequate amount of recovery time was permitted between 1RM trials (3 - 5 min) [20]. MVC of the elbow extensors (right arm) was measured by using an isokinetic dynamometer (Biodex System 3, Biodex Medical Systems Inc., Shirley, NY, USA). The subjects were comfortably seated on a chair and the arm was positioned on a firm and stable table at chest level with an elbow joint angle of $90^{\circ}\left(0^{\circ}\right.$ at full extension). The upper arm was maintained in the horizontal plane while the subject's wrist was fixed at the end of the lever arm in a position halfway between supination and pronation. The elbow extensor force was measured with a transducer, while a diagonal strap was secured over the elbow to maintain a stationary position during the MVC. Subjects were instructed to contract as fast and forcefully as possible. MVC was measured twice. If MVC torque for the first two MVCs varied by $>5 \%$, up to two additional MVCs were performed. Each effort was held for $\sim 5 \mathrm{~s}$. The coefficient of variation (CV) for this measurement from test to retest was 3.1\% [21]. Both MVC and 1RM tests (same day and about 20 min apart between two tests) were performed before training and after 3 and 6 weeks of training.

\subsection{Muscle Size Measurements}

Multi-slice MRI images of the upper arm and chest were obtained using a MRI scanner (General Electric Yokogawa Signa 0.2-T, Milwaukee, WI, USA). A T1-weighted, spin-echo, axial plane sequence was performed with a 520 ms repetition time and a $20 \mathrm{~ms}$ echo time. Subjects rested quietly in the magnet bore in a supine position with their arms extended. The lateral epicondyle of the humerus was used as the origin point, and continuous transverse images with $1.0 \mathrm{~cm}$ slice thickness $(0.2 \mathrm{~cm}$ interslice gap) were obtained from the lateral epicondyle of the humerus to the acromial process of the scapula for each subject (Figure 1). All MRI data were transferred to a personal computer for analysis using specially designed image analysis software (TomoVision Inc., Montreal, Canada). For each slice, skeletal muscle tissue crosssectional area (CSA) was digitized. Triceps brachii (TB) and pectoralis major (PM) muscle CSA of 3 continuous slices for the muscle belly were averaged to represent a single data point for statistical analysis, respectively. We have previously determined that the $\mathrm{CV}$ of this measurement was less than 1\% [21].

\subsection{Statistical Analysis}

All values are expressed as mean [SD]. TB and PM muscle CSA, 1RM, MVC data were analyzed using two-way ANOVA with repeated measures (group $\times$ time). Post hoc testing was performed using Tukey-Kramer when appropriate. Pre-training values of each training protocol were compared using a paired $t$-test. Pearson productmoment correlation coefficients determined the association between high-load and low-load hypertrophy changes in TB and PM muscle CSA. Significance was set at $p<$ 0.05. All analyses were performed using JMP statistical software version 8.0 (SAS Institute, Cary, NC, USA).

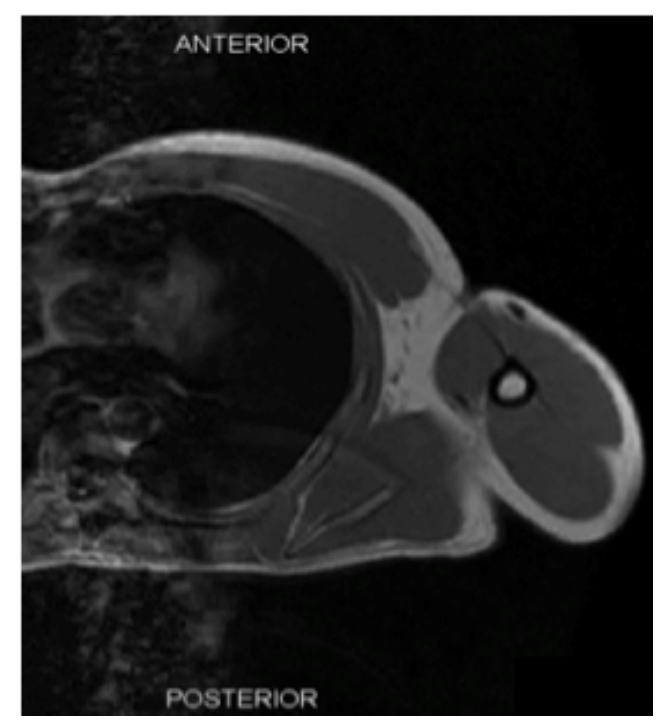

Figure 1. Typical magnetic resonance imaging image showing transverse scan of the chest. 


\section{Results}

There was no difference in body weight at pre-training between HL-RT (68.9 [8.1] kg) and LL-RT (68.8 [8.0] $\mathrm{kg})$. After 6-week of training, body weight increased ( $p<$ 0.05 ) by $0.6 \mathrm{~kg}$ in the HL-RT and $0.6 \mathrm{~kg}$ in the LL-RT. During the LL-RT protocol, the average total number of repetitions for each exercise session was 141 [14].

Following 6 weeks of training, $1 \mathrm{RM}$ and MVC strength increased $(p<0.05)$ significantly in both HL-RT and LL-RT protocols. However, the percent increases in strength were lower $(p<0.05)$ in the LL-RT $(1$ RM 8.6 [2.9]\%, MVC 6.5 [4.9]\%) than in the HL-RT (1 RM 21.0 [5.9]\%, MVC 13.9 [7.5]\%) (Figure 2). Before the start of the LL-RT, 1-RM and MVC strength had not returned

(a)

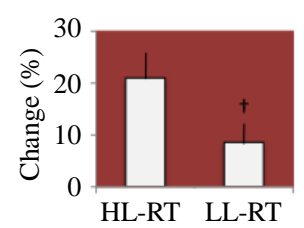

to pre-training HL-RT 1-RM and MVC strength levels

\section{(Figure 2).}

At the start of training, muscle CSA in the PM was the same between the HL-RT and LL-RT protocols, whereas muscle CSA in the TB was $2.2 \%$ higher $(p=0.03)$ in LL-RT than in HL-RT. The TB muscle CSA increased ( $p$ $<0.01$ ) following LL-RT and HL-RT and the percent increase in muscle CSA was similar between the two training protocols (LL-RT 9.8 [4.6]\%, HL-RT 11.9 [2.6]\%) (Figure 3(a)). Similarly, absolute and relative increases $(p<0.01)$ in PM muscle CSA were similar between HL-RT and LL-RT (Figure 3B). A significant correlation was observed between percent increase in muscle CSA following HL-RT and LL-RT in the TB and PM muscles (Figure 4).

(b)
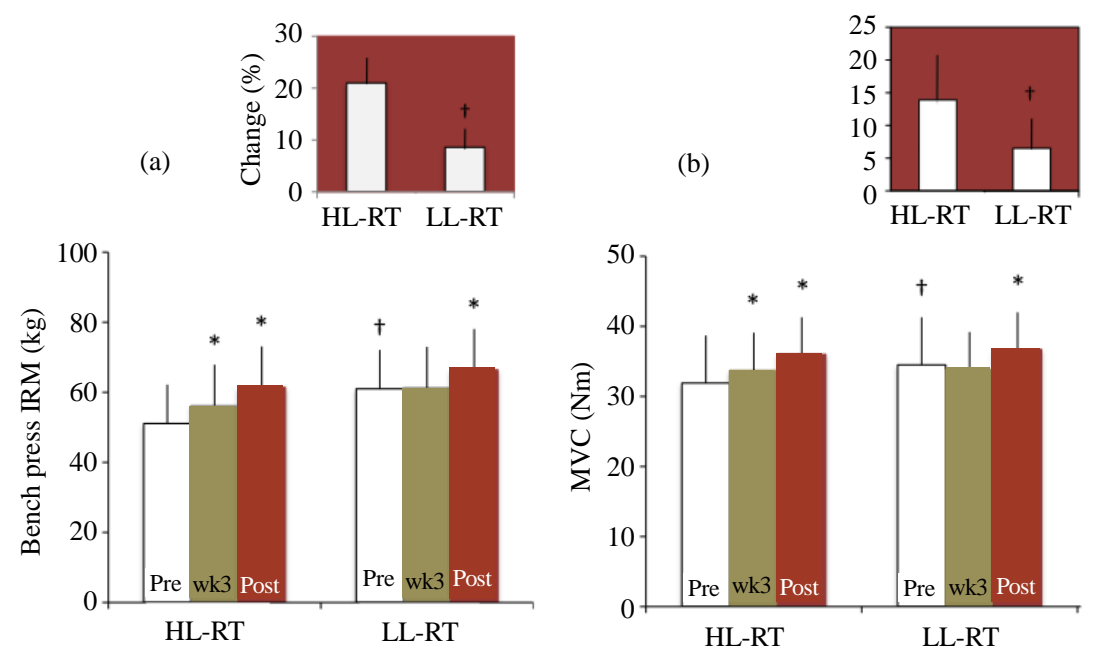

(a)

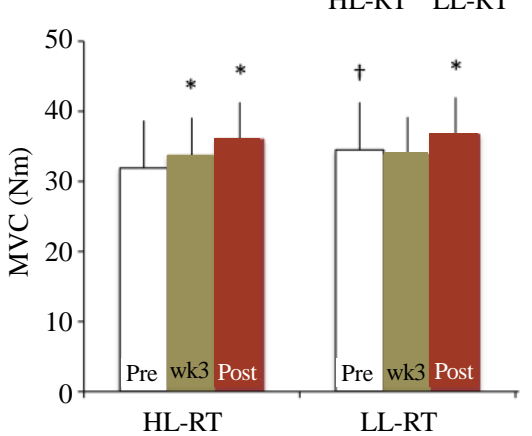

(b)

Figure 2. Changes in maximum dynamic (bench press one repetition maximum) and isometric (elbow extension) strength following 6 weeks of high-load (HL-RT) and low-load (LL-RT) resistance training. Pre, before training; wk3, after 3 weeks; Post, after 6 weeks. ${ }^{*} p<0.05$ vs. pre- training, ${ }^{\dagger} p<0.05$ vs. HL-RT.

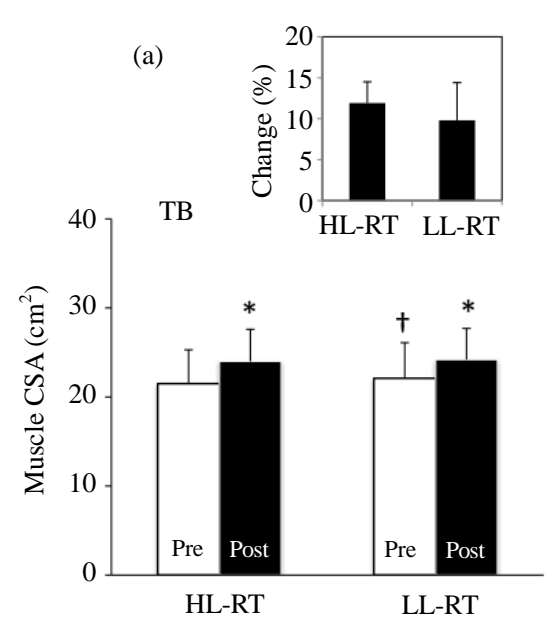

(a)

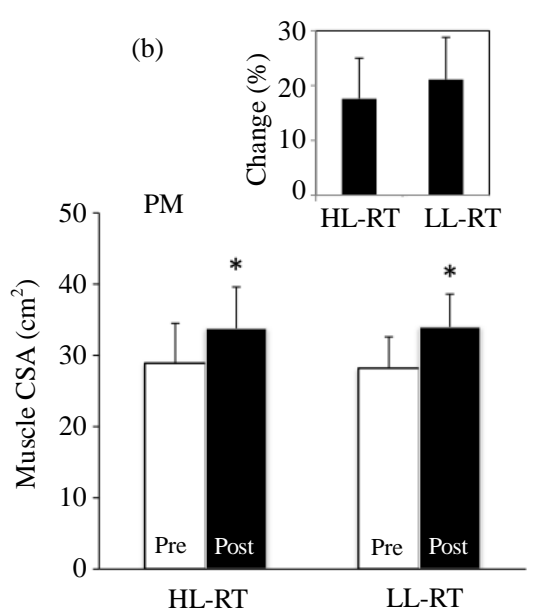

(b)

Figure 3. Changes in muscle cross-sectional area (CSA) in the triceps brachii (TB) and pectoralis major (PM) muscles following 6 weeks of high-load (HL-RT) and low-load (LL-RT) resistance training. Pre, before training; Post, after 6 weeks. ${ }^{*} p<$ 0.05 vs. pre-training, ${ }^{\dagger} p<0.05$ vs. HL-RT. 

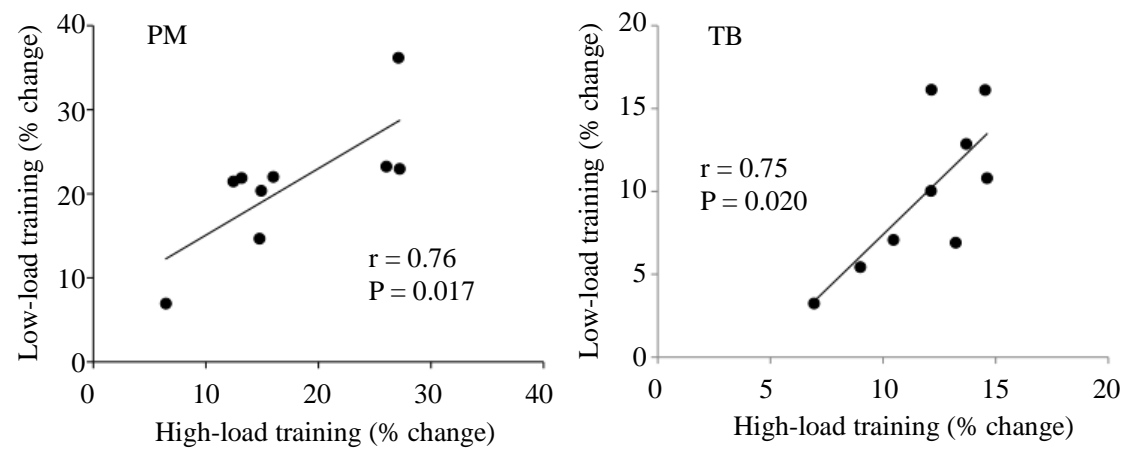

Figure 4. Relationship between percent increase in muscle cross-sectional area following 6 weeks of high-load (HL-RT) and low-load (LL-RT) resistance training in the triceps brachii (TB) and pectoralis major (PM) muscles.

\section{Discussion}

This study found that 1) LL-RT to volitional fatigue and HL-RT results in similar levels of skeletal muscle hypertrophy in the upper body and 2) significant correlations in the degree of muscle hypertrophy between LL-RT to volitional fatigue and HL-RT. This data suggests that skeletal muscle hypertrophy can occur independent of a higher load in the upper body as long as there is adequate exercise volume. In addition, one year of detraining from HL-RT results in a complete loss of muscle size, however muscle strength was decreased but still elevated above the pre-training level.

\subsection{Muscle Hypertrophy}

Six weeks of high-load (75\% $1 \mathrm{RM}$ ) resistance training resulted in significant skeletal muscle hypertrophy. Interestingly, after 12 months of detraining the same subjects then performed low-load resistance training to volitional fatigue and found similar increases in skeletal muscle hypertrophy compared to that observed with high-load training. This is contrary to previous research $[9,10]$ and recommendations [7] that report higher-loads to be superior. However, the research in which those recommendations were largely based were matched for work and it appears that in order for low-loads to increase muscle hypertrophy to levels similar to high-loads, exercise must be taken to volitional fatigue [5].

This study confirms acute research from Burd et al. [8] who found similar increases in myofibril MPS independent of exercise load when exercise was taken to volitional fatigue. This might be related to the significant increase in muscle time under tension when repetitions are taken to volitional fatigue as this has recently been found to be an important variable in the synthetic response [2]. In addition, MPS from resistance training occurs primarily from the activation of signaling proteins, primarily S6K1, which are approximately 3 to 4 -fold higher in FT fibers compared to ST [22]. Furthermore, phosphorylation of this signaling protein has shown to be predictive of skeletal muscle hypertrophy [23]. This suggests that skeletal muscle hypertrophy occurs independent of a higher exercise load, as long as FT fibers are activated from sufficient exercise volume [5,6]. It is acknowledged that the protein degradation response to low-load resistance training to volitional fatigue is not known, as research is typically completed under the assumption that synthesis rates and not degradation rates are more responsive to resistance exercise in healthy humans [24]. The similar levels of muscle hypertrophy between protocols suggest that this assumption is likely true for the upper body. This also supports recent research completed in the lower body, which found significant muscle hypertrophy with low load (30\% 1 RM) knee extensor exercise to fatigue [16].

Interestingly, it should be mentioned that rodent data suggest that the myonuclei gained from resistance training are not lost following 3 months of detraining [25]. This has led some to speculate that this retention of myonuclei is important in the "muscle memory" response to exercise. Therefore, if one is trained following the cessation of training, it might be possible that the rebound in muscle hypertrophy is due to the myonuclei that were added with training and maintained through muscle atrophy. It is currently unknown how this translates to humans or how long this effect lasts, but we cannot rule out the possibility that this may be playing some role in the equal response between variables.

The percentage increases in muscle hypertrophy for the TB and PM were larger than what has been previously reported for the lower body. Unfortunately, the molecular mechanisms for upper body muscle hypertrophy are currently under studied when compared with what is known for the lower body. However, the results of the present investigation suggest that heavy resistance exercise induced activation of muscle protein metabolism may be more responsive in the upper body compared to the lower body. To illustrate, Seynnes et al. [26] observed a 7\% increase in quadriceps femoris CSA following 35 days of lower body bilateral knee extensions. In 
addition, Abe et al. [20] observed after a 6 week total body workout $(70 \% 1 \mathrm{RM})$, that the quadriceps muscle thickness increased 5\%, however the PM and TB increased $13 \%$ and $9 \%$, respectively. Furthermore, using a MRI, muscle CSA increased $16 \%$ in the PM and $10 \%$ in the TB following 18 days of bench press training (75\% 1RM) [27]. Yasuda et al. [28] also observed that 18 days of bench press training ( $75 \% 1 \mathrm{RM}$ ) resulted in an $18 \%$ increase in PM and a $10 \%$ increase in the TB. The current findings are in agreement with the previous research in the upper body which suggests that the upper body may have a higher capacity for muscle hypertrophy than the lower body.

\subsection{Muscular Strength}

Changes in strength between the LL-RT and HL-RT are another interesting finding from this study. Both groups had significant increases in strength following training; however, the percent increases in strength were significantly lower in the LL-RT protocol. An explanation of the smaller relative increases in strength may be due to the fact that detraining after HL-RT did not cause strength values to return to baseline levels thereby producing smaller changes in strength. Although subjects were told to return back to their pre-training lifestyle, it is possible that subjects maintained a level of activity high enough to maintain strength but not muscle mass. Further, it is possible that the neural adaptation to resistance exercise is longer lasting than the hypertrophic response. Indeed, there is evidence to support the finding that strength does not return to baseline levels despite detraining. In young women who did 20 weeks of strength training and then detrained for 30 - 32 weeks, strength levels significantly decreased but did not return to pretraining levels [29]. In addition, Bickel et al. [30] found that after 16 weeks of lower body training and 32 weeks of detraining that strength significantly decreased by $7 \%$ but remained 23\% above baseline. Another study in older adults found that 2 years of training followed by 3 years of detraining produced significant decreases in dynamic strength but levels remained slightly above baseline values and significantly higher than control subjects [31]. Although the reasons for this maintenance of strength are unknown from the present investigation, it is possible that following detraining there was a partial maintenance of the increased volitional drive from the supraspinal center which may have maintained part of the increased muscle activation likely gained from HL-RT [32]. Therefore, the lower amounts of strength observed in the LL-RT group compared to the HL-RT may be more a function of the training effect rather than the intervention itself. Also, all subjects began training with high-load resistance training and finished with low-load training, therefore it remains unknown if the same strength effects would be observed if the protocols were reversed. Lastly, an alternative explanation is that the specificity of training may dictate the overall maximal gains in strength. For example, the results may suggest that the consistent practice of lifting a heavy load is necessary to maximize gains in muscular strength of the trained movement.

\section{Conclusion}

This study verifies that similar degrees of muscle hypertrophy can occur in the upper body independent of a high external load, provided enough muscular work is completed. This data seems to support that the acute myofibril MPS responses previously observed with LL-RT to fatigue do translate to chronic training adaptation. These results demonstrate that significant muscle hypertrophy can occur without high-load resistance training and suggests that the focus on percentage of external load as the important deciding factor on muscle adaptation (i.e. muscle hypertrophy) is too simplistic and inappropriate.

\section{Acknowledgements}

The authors thank the students who participated in this study. None of the authors had financial or personal conflict of interest with regard to this study. No sources of funding were used to assist in the preparation of this manuscript.

\section{REFERENCES}

[1] M. J. Drummond, C. S. Fry, E. L. Glynn, K. L. Timmerman, J. M. Dickinson, D. K. Walker, D. M. Gundermann, E. Volpi and B. B. Rasmussen, "Skeletal Muscle Amino Acid Transporter Expression Is Increased in Young and Older Adults Following Resistance Exercise,” Journal of Applied Physiology, Vol. 111, No. 1, 2011, pp. 135-142. doi:10.1152/japplphysiol.01408.2010

[2] N. A. Burd, R. J. Andrews, D. W. West, J. P. Little, A. J. Cochran, A. J. Hector, J. G. Cashaback, M. J. Gibala, J. R. Potvin, S. K. Baker and S. M. Phillips, "Muscle Time under Tension during Resistance Exercise Stimulates Differential Muscle Protein Sub-Fractional Synthetic Responses in Men,” Journal of Physiology, Vol. 590, No. 2, 2012, pp. 351-362.

[3] G. Adams, "The Molecular Response of Skeletal Muscle to Resistance Training,” Deutsche Zeitschrift Sportmedizin, Vol. 61, No. 3, 2010, pp. 61-67.

[4] D. Wagner, "Skeletal Muscle Growth: Hypertrophy and Hyperplasia," Strength and Conditioning Journal, Vol. 18, No. 5, 1996, pp. 38-39. doi:10.1519/1073-6840(1996)018<0038:SMGHAH>2.3. $\mathrm{CO} ; 2$

[5] J. P. Loenneke, C. A. Fahs, J. M. Wilson and M. G. Bemben, "Blood Flow Restriction: The Metabolite/Volume Threshold Theory,” Medical Hypotheses, Vol. 77, No. 5, 
2011, pp. 748-752. doi:10.1016/j.mehy.2011.07.029

[6] S. M. Phillips, "Physiologic and Molecular Bases of Muscle Hypertrophy and Atrophy: Impact of Resistance Exercise on Human Skeletal Muscle (Protein and Exercise Dose Effects)," Applied Physiology, Nutrition, and Metabolism, Vol. 34, No. 3, 2009, pp. 403-410. doi:10.1139/H09-042

[7] American College of Sports Medicine (ACSM) Position Stand, "Progression Models in Resistance Training for Healthy Adults," Medicine and Science in Sports and Exercise, Vol. 41, No. 3, 2009, pp. 687-708. doi:10.1249/MSS.0b013e3181915670

[8] N. A. Burd, D. W. West, A. W. Staples, P. J. Atherton, J. M. Baker, D. R. Moore, A. M. Holwerda, G. Parise, M. J. Rennie, S. K. Baker and S. M. Phillips, "Low-Load High Volume Resistance Exercise Stimulates Muscle Protein Synthesis More than High-Load Low Volume Resistance Exercise in Young Men,” PLoS One, Vol. 5, No. 8, 2010, e12033. doi:10.1371/journal.pone.0012033

[9] J. M. Willardson, "The Application of Training to Failure in Periodized Multiple-Set Resistance Exercise Programs,” Journal of Strength and Conditioning Research, Vol. 21, No. 2, 2007, pp. 628-631.

[10] G. E. Campos, T. J. Luecke, H. K. Wendeln, K. Toma, F. C. Hagerman, T. F. Murray, K. E. Ragg, N. A. Ratamess, W. J. Kraemer and R. S. Staron, "Muscular Adaptations in Response to Three Different Resistance-Training Regimens: Specificity of Repetition Maximum Training Zones,” European Journal of Applied Physiology, Vol. 88, No. 1-2, 2002, pp. 50-60. doi:10.1007/s00421-002-0681-6

[11] B. Leger, R. Cartoni, M. Praz, S. Lamon, O. Deriaz, A. Crettenand, C. Gobelet, P. Rohmer, M. Konzelmann, F. Luthi and A. P. Russell, "Akt Signalling through GSK3Beta, mTOR and Foxo1 Is Involved in Human Skeletal Muscle Hypertrophy and Atrophy," Journal of Physiology, Vol. 576, No. 3, 2006, pp. 923-933. doi:10.1113/jphysiol.2006.116715

[12] D. W. West, N. A. Burd, J. E. Tang, D. R. Moore, A. W. Staples, A. M. Holwerda, S. K. Baker and S. M. Phillips, "Elevations in Ostensibly Anabolic Hormones with Resistance Exercise Enhance neither Training-Induced Muscle Hypertrophy nor Strength of the Elbow Flexors," Journal of Applied Physiology, Vol. 108, No. 1, 2010, pp. 60-67. doi:10.1152/japplphysiol.01147.2009

[13] D. W. West and S. M. Phillips, “Associations of Exercise-Induced Hormone Profiles and Gains in Strength and Hypertrophy in a Large Cohort after Weight Training," European Journal of Applied Physiology, Vol. 112, No. 7, 2012, pp. 2693-2702. doi:10.1007/s00421-011-2246-Z

[14] V. G. Coffey, Z. Zhong, A. Shield, B. J. Canny, A. V. Chibalin, J. R. Zierath and J. A. Hawley, "Early Signaling Responses to Divergent Exercise Stimuli in Skeletal Muscle from Well-Trained Humans," FASEB Journal, Vol. 20, No. 1, 2006, pp. 190-192.

[15] S. B. Wilkinson, M. A. Tarnopolsky, E. J. Grant, C. E. Correia and S. M. Phillips, "Hypertrophy with Unilateral Resistance Exercise Occurs without Increases in Endogenous Anabolic Hormone Concentration,” European
Journal of Applied Physiology, Vol. 98, No. 6, 2006, pp. 546-555. doi:10.1007/s00421-006-0300-Z

[16] C. J. Mitchell, T. A. Churchward-Venne, D. W. West, N. A. Burd, L. Breen, S. K. Baker and S. M. Phillips, "Resistance Exercise Load Does Not Determine Training-Mediated Hypertrophic Gains in Young Men," Journal of Applied Physiology, Vol. 113, No. 1, 2012, pp. 71-77. doi:10.1152/japplphysiol.00307.2012

[17] R. Ogasawara, R. S. Thiebaud, J. P. Loenneke and T. Abe, "Time Course for Arm and Chest Muscle Thickness Changes Following Bench Press Training,” Interventional Medicine and Applied Science, Vol. 4, No. 4, 2012, pp. 217-220. doi:10.1556/IMAS.4.2012.4.7

[18] R. Ogasawara, T. Yasuda, N. Ishii and T. Abe, "Comparison of Muscle Hypertrophy Following 6-Month of Continuous and Periodic Strength Training," European Journal of Applied Physiology, in Press. doi:10.10007/s00421-012-2511-9

[19] P. Kannus, D. Alosa, L. Cook, R. J. Johnson, P. Renstrom, M. Pope, B. Beynnon, K. Yasuda, C. Nichols and M. Kaplan, "Effect of One-Legged Exercise on the Strength, Power and Endurance of the Contralateral Leg. A Randomized, Controlled Study Using Isometric and Concentric Isokinetic Training," European Journal of Applied Physiology and Occupational Physiology, Vol. 64, No. 2, 1992, pp. 117-126. doi:10.1007/BF00717948

[20] T. Abe, D. V. DeHoyos, M. L. Pollock and L. Garzarella, "Time Course for Strength and Muscle Thickness Changes Following Upper and Lower Body Resistance Training in Men and Women," European Journal of Applied Physiology, Vol. 81, No. 3, 2000, pp. 174-180. doi:10.1007/s004210050027

[21] T. Yasuda, R. Ogasawara, M. Sakamaki, H. Ozaki, Y. Sato and T. Abe, "Combined Effects of Low-Intensity Blood Flow Restriction Training and High-Intensity Resistance Training on Muscle Strength and Size," European Journal of Applied Physiology, Vol. 111, No. 10, 2011, pp. 2525-2533. doi:10.1007/s00421-011-1873-8

[22] J. Tannerstedt, W. Apro and E. Blomstrand, "Maximal Lengthening Contractions Induce Different Signaling Responses in the Type I and Type II Fibers of Human Skeletal Muscle," Journal of Applied Physiology, Vol. 106, No. 4, 2009, pp. 1412-1418. doi:10.1152/japplphysiol.91243.2008

[23] G. Terzis, G. Georgiadis, G. Stratakos, I. Vogiatzis, S. Kavouras, P. Manta, H. Mascher and E. Blomstrand, "Resistance Exercise-Induced Increase in Muscle Mass Correlates with p70S6 Kinase Phosphorylation in Human Subjects,” European Journal of Applied Physiology, Vol. 102, No. 2, 2008, pp. 145-152. doi:10.1007/s00421-007-0564-y

[24] M. J. Rennie, H. Wackerhage, E. E. Spangenburg and F. W. Booth, "Control of the Size of the Human Muscle Mass,” Annual Review of Physiology, Vol. 66, 2004, pp. 799-828. doi:10.1146/annurev.physiol.66.052102.134444

[25] J. C. Bruusgaard, I. B. Johansen, I. M. Egner, Z. A. Rana and K. Gundersen, "Myonuclei Acquired by Overload Exercise Precede Hypertrophy and are Not Lost on Detraining," Proceedings of the National Academy of Sci- 
ences in the United States of America, Vol. 107, No. 34, 2010, pp. 15111-15116. doi:10.1073/pnas.0913935107

[26] O. R. Seynnes, M. de Boer and M. V. Narici, "Early Skeletal Muscle Hypertrophy and Architectural Changes in Response to High-Intensity Resistance Training,” Journal of Applied Physiology, Vol. 102, No. 1, 2007, pp. 368-373. doi:10.1152/japplphysiol.00789.2006

[27] R. Ogasawara, T. Yasuda, M. Sakamaki, H. Ozaki and T. Abe, "Effects of Periodic and Continued Resistance Training on Muscle CSA and Strength in Previously Untrained Men," Clinical Physiology and Functional Imaging, Vol. 31, No. 5, 2011, pp. 399-404. doi:10.1111/i.1475-097X.2011.01031.x

[28] T. Yasuda, R. Ogasawara, M. Sakamaki, M. G. Bemben and T. Abe, "Relationship between Limb and Trunk Muscle Hypertrophy Following High-Intensity Resistance Training and Blood Flow-Restricted Low-Intensity Resistance Training," Clinical Physiology and Functional Imaging, Vol. 31, No. 5, 2011, pp. 347-351. doi:10.1111/j.1475-097X.2011.01022.X

[29] R. S. Staron, M. J. Leonardi, D. L. Karapondo, E. S. Malicky, J. E. Falkel, F. C. Hagerman and R. S. Hikida,
"Strength and Skeletal Muscle Adaptations in HeavyResistance-Trained Women after Detraining and Retraining,” Journal of Applied Physiology, Vol. 70, No. 2, 1991, pp. 631-640.

[30] C. S. Bickel, J. M. Cross and M. M. Bamman, "Exercise Dosing to Retain Resistance Training Adaptations in Young and Older Adults," Medicine and Science in Sports Exercise, Vol. 43, No. 7, 2011, pp. 1177-1187. doi:10.1249/MSS.0b013e318207c15d

[31] K. Smith, K. Winegard, A. L. Hicks and N. McCartney, "Two Years of Resistance Training in Older Men and Women: The Effects of Three Years of Detraining on the Retention of Dynamic Strength," Canadian Journal of Applied Physiology, Vol. 28, No. 3, 2003, pp. 462-474. doi:10.1139/h03-034

[32] C. Del Balso and E. Cafarelli, "Adaptations in the Activation of Human Skeletal Muscle Induced by Short-Term Isometric Resistance Training,” Journal of Applied Physiology, Vol. 103, No. 1, 2007, pp. 402-411. doi:10.1152/japplphysiol.00477.2006 